\title{
REVISION 2
}

\section{Morin-type transition in 5C pyrrhotite}

10 We report the discovery of a low temperature spin-flop transition in 5C pyrrhotite at $\sim 155 \mathrm{~K}$

11 that is similar to those seen in hematite at $260 \mathrm{~K}$ and $\mathrm{FeS}$ (troilite) at $440 \mathrm{~K}$. The $5 \mathrm{C}$ crystal was

12 produced by annealing a $4 \mathrm{C}$ pyrrhotite crystal at $875 \mathrm{~K}$, to produce a change in the vacancy-

13 ordering scheme that developed during cooling. The 5C structure is confirmed by single crystal x-

14 ray diffraction and the stoichiometry and homogeneity by electron microprobe and SEM BSE

15 mapping. RUS, heat capacity and magnetisation measurements from room temperature down to 2

$16 \mathrm{~K}$ are reported. The transition is marked by a steep change in elastic properties at the transition

17 temperature, a peak in the heat capacity and weak anomalies in measurements of magnetisation.

18 Magnetic hysteresis loops and comparison with the magnetic properties of 4C pyrrhotite suggest

19 that the transition involves a change in orientation of moments between two different

20 antiferromagnetic structures, perpendicular to the crystallographic $c$-axis at high temperatures and

21 parallel to the crystallographic $c$-axis at low temperatures. The proposed structures are consistent 
22 with a group theoretical treatment that also predicts a first order transition between the magnetic 23 structures. 


\section{I. INTRODUCTION}

25 The pyrrhotite system comprises a number of related structures having compositions $\mathrm{Fe}_{1-x} \mathrm{~S}$

26 where $0<x<0.125$. The best known of these structures are troilite $(\mathrm{FeS})$ and $4 \mathrm{C}$ pyrrhotite

$27\left(\mathrm{Fe}_{7} \mathrm{~S}_{8}\right) .4 \mathrm{C}$ is so called because it is a superstructure with a 4 cell repeat along the $c$-axis with

28 respect to the parent NiAs structure (Bertaut 1953; Powell 2004). Other commensurate

29 superstructures have been reported as 3C (Fleet 1971, Nakano et al 1979), 5C (de Villiers et al

30 2009, Elliot 2010, Liles and de Villiers 2012) and 6C (Koto et al 1975, de Villiers and Liles 2010),

31 while some are incommensurate (Nakazawa and Morimoto 1971, Morimoto et al 1975, Yamamoto

32 and Nakazawa 1982, Izaola et al 2007). Ferrimagnetic pyrrhotite (4C) is an important carrier of

33 magnetic remanence on earth and possibly also on Mars (e.g. Martin-Hernandez et. al 2008;

34 Rochette et. al 2005), but 4C structures are not unique and the other superstructure types have

35 different structure/property relationships which are much less well known. The key issue in

36 relation to the magnetic structures are how vacancies order on the cation sites as the stoichiometry

37 changes and how the distribution of vacancies then controls the magnetic structures.

38 By adopting a group theoretical approach, Haines et al. (2019a) have shown that the

39 commensurate superstructures of pyrrhotite, which have different Fe/vacancy ordering schemes,

40 are all likely to undergo a magnetically driven spin reorientation transition with accompanying

41 small distortions of the crystal lattice at low temperatures. The prediction for the case of $5 \mathrm{C}$

42 pyrrhotite is for an abrupt spin-flop transition similar to the Morin transition in hematite. The

43 present work focuses on the magnetic and elastic properties of a natural $4 \mathrm{C}$ crystal that transformed

44 unexpectedly to 5C crystal after being heated above its concomitant vacancy ordering and Néel

45 temperature of $595 \mathrm{~K}$. We report a newly identified magnetic transition at $\sim 155 \mathrm{~K}$ in the $5 \mathrm{C}$ crystal

46 and propose that it is a spin-flop transition between two antiferromagnetic structures, with an 
47 origin that is closely related to that of the magnetic transitions already known in FeS at $440 \mathrm{~K}$ and $484 \mathrm{C} \mathrm{Fe}_{7} \mathrm{~S}_{8}$ at $35 \mathrm{~K}$.

49 In general, 5C pyrrhotite is found to have a composition close to $\mathrm{Fe}_{9} \mathrm{~S}_{10}$. However, Pósfai et.

50 al. (2000) reported a 5C polytype with composition $\mathrm{Fe}_{7} \mathrm{~S}_{8}$. Kontny et. al. (2000) also presented

51 some x-ray diffraction and magnetisation measurements on multiphase samples, suggesting the

52 possibility of pyrrhotite with a $5 \mathrm{C}$ structure having the stoichiometry close to the $\mathrm{Fe}_{7} \mathrm{~S}_{8}$ that is

53 usually associated with the 4C structure. De Villiers et al. (2009) proposed space group number

$5463 \mathrm{Cmce}$ (formerly $\mathrm{Cmca}$ ) as the correct crystallographic space group at room temperature. Elliot

55 (2010) had claimed $P 22_{1} / c$ for the room temperature structure. Liles and de Villiers (2012)

56 subsequently proposed $P 2{ }_{1}$ for the structure at both room temperature and $120 \mathrm{~K}$. There do not

57 appear to have been any studies of the magnetic structure.

58 In this work, we first establish the stoichiometry of the sample through electron microprobe

59 analysis before going on to present a structural solution in the space group 63 Cmce based on

60 single crystal diffraction data collected on a single crystal sample of 5C pyrrhotite at room

61 temperature. We then present heat capacity, DC magnetisation and resonant ultrasound

62 spectroscopy (RUS) measurements on a second single crystal over the range 2-300 K.

\section{II. SAMPLE PREPARATION}

64 The single crystal sample of pyrrhotite used in this study was cut from a cm-sized crystal in the 65 mineral collection of the South Australia Museum that had originated from a mine in Mexico.

66 Samples from the same parent crystal have been described in Haines et al (2019c). At the start, it 67 was nearly in the shape of a rectangular parallelepiped with two pairs of parallel faces $1.7 \mathrm{~mm}$ and $681.5 \mathrm{~mm}$ apart and a third pair of non-parallel faces separated by between $1 \mathrm{~mm}$ and $1.5 \mathrm{~mm}$. The 69 first experiment was to collect RUS spectra through the temperature interval 10 - $295 \mathrm{~K}$ to confirm 
70 that it was 4C pyrrhotite with a Besnus transition (Besnus and Meyer 1964) at 35 K (see Haines

71 et al. 2019b).

72 After removal from the RUS instrument, the crystal was heated slowly to $875 \mathrm{~K}$ in flowing

73 argon, with an oxygen trap, over four and a half days before cooling it back to room temperature

74 over three days. The sample was held between two alumina rods. The heating and cooling rates

75 through the $595 \mathrm{~K}$ transition were $2.2 \mathrm{~K} /$ hour. As a consequence of this treatment, the crystal

76 acquired a thin oxide layer that was removed mechanically, and also broke into two pieces. The

77 larger piece had a mass of $9.1 \pm 0.1 \mathrm{mg}$ and was used for heat capacity, magnetisation and RUS

78 measurements. On completion of these measurements, the larger piece was mounted and

79 polished for chemical analysis. The smaller piece weighed $2.2 \pm 0.1 \mathrm{mg}$ and was broken up for

80 the single crystal diffraction measurements.

81 III. ELECTRON MICROPROBE ANALYSIS

82 Chemical analysis on the larger piece was carried out using a Cameca SX100 electron

83 microprobe in the Department of Earth Sciences, University of Cambridge (instrumental

84 conditions: $20 \mathrm{keV}, 10 \mathrm{nA}, 1 \mu \mathrm{m}$ beam diameter). With respect to 10 sulphur atoms, the average

85 of 20 analyses gave the number of Fe atoms as $9.21 \pm 0.05 . \mathrm{Co}, \mathrm{Cu}, \mathrm{Mn}, \mathrm{Ni}$ and $\mathrm{Zn}$ were measured

86 for, but all were below the detection limit on all of the 20 analysis spots. We highlight here that

87 this shows a significant change in stoichiometry from the parent crystal described in Haines et al.

88 (2019c) in which electron microprobe analysis revealed a homogeneous and pure 4C crystal of

89 stoichiometry 7.00(6) Fe atoms per 8 sulphur atoms. We suggest that this change of stoichiometry

90 is due to the loss of sulphur from the crystal rather than the addition of iron for which no source

91 or mechanism is present. To check for zoning or inhomogeneity in the stoichiometry of the crystal

92 a scanning electron microscope back-scattered electron (SEM-BSE) analysis map of nearly the

93 whole surface of the same piece used for the electron microprobe analysis was collected. The 
94 SEM-BSE map showed no sign of any chemical inhomogeneity above the level of detection. We

95 therefore conclude that the transformation from stoichiometry $\mathrm{Fe}_{7.00(6)} \mathrm{S}_{8}$ to $\mathrm{Fe} \mathrm{g.21(5)} \mathrm{S}_{10}$ is

96 complete and homogeneous. The images are included as supplemental information (SI 2020).

\section{IV. SINGLE CRYSTAL X-RAY DIFFRACTION}

99 The single crystal used for X-ray diffraction was black and plate-shaped, with dimensions

$100 \sim 0.080 \times 0.040 \times 0.020 \mathrm{~mm}^{3}$. It was mounted on a MITIGEN holder in perfluoroether oil on a

101 Rigaku FRE+ diffractometer equipped with VHF Varimax confocal mirrors and an AFC12

102 goniometer and HyPix $6000 \mathrm{HE}$ detector. The temperature was $293 \pm 2 \mathrm{~K}$ during scans of $0.5^{\circ}$ per

103 frame for $1.3 \mathrm{~s}$ with Mo K radiation (Rotating-anode X-ray tube, $40 \mathrm{kV}, 30 \mathrm{~mA}$ ). The total number

104 of runs and images was based on the strategy calculation from the program CrysAlisPro (Rigaku,

105 V1.171.39.46, 2018). The maximum resolution achieved was at $27.478^{\circ}(0.77 \AA)$. Cell parameters

106 were retrieved using the CrysAlisPro (Rigaku, V1.171.39.46, 2018) software and refined using

107 CrysAlisPro (Rigaku, V1.171.39.46, 2018) on 5787 reflections, $22 \%$ of the observed reflections.

108 Data reduction, scaling and absorption corrections were performed using CrysAlisPro (Rigaku,

$109 \mathrm{~V} 1.171 .39 .46,2018)$. The final completeness is $99.7 \%$ out to $25^{\circ}$ in $\Theta$. A multi-scan absorption

110 correction was performed using CrysAlisPro 1.171.39.46 (Rigaku Oxford Diffraction, 2018) using

111 spherical harmonics as implemented in SCALE3 ABSPACK. The absorption coefficient of this

112 material is $13.664 \mathrm{~mm}^{-1}$ at this wavelength $(=0.711 \AA)$ and the minimum and maximum

113 transmissions are 0.522 and 1.000 .

114 Figure 1 shows a section of the $(0 \mathrm{kl})$ plane of the diffraction pattern, with 5 superlattice

115 reflections clearly visible along the $c^{*}\left(\mathrm{k}_{\mathrm{z}}\right)$ of the parent NiAs cell. We looked for evidence of

116 incommensuration by initially indexing the pattern with the NiAs unit cell and then using an

117 incommensurate $\mathrm{k}$-vector to describe the remaining peaks. The k-vector which describes the 
118 pattern has components of 0.500(3) along the planar hexagonal axes and 0.204(6) perpendicular

119 to the ab-plane of the parent structure. This is therefore commensurate within experimental 120 uncertainty.

121

122

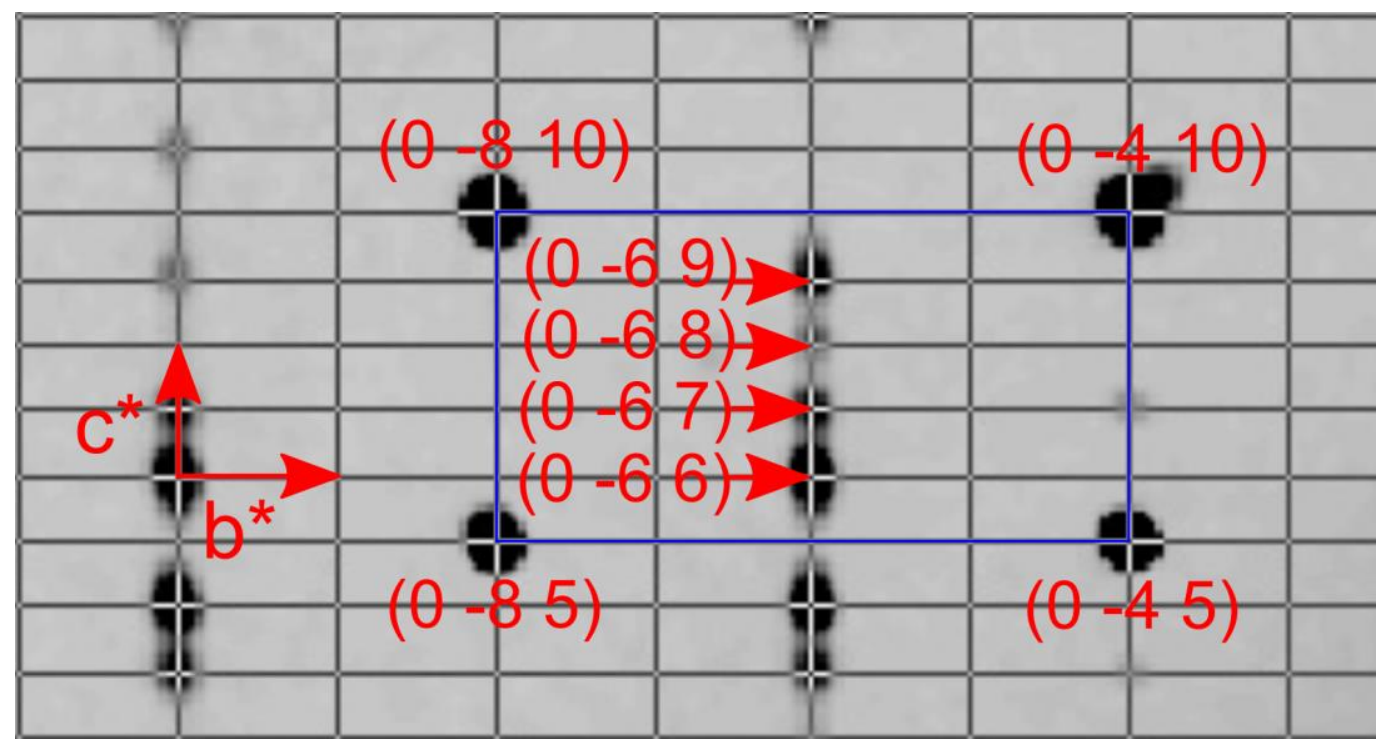

123 Figure 1. Part of the $(0 \mathrm{kl})$ precession image of the room temperature single crystal X-ray

124 diffraction pattern, showing the five-fold superlattice reflections of the $5 \mathrm{C}$ structure. The data has

125 been reduced using the cell from the Cmce structure and the peaks are labelled with the $(h k l)$ of

126 the Cmce structure. The direction of the $b^{*}$ and $c^{*}$ are given in red on the left. The unit cell of the

127 Cmce structure is shown by the grey grid. The projection of the hexagonal parent cell onto the

128 Cmce $(0 \mathrm{kl})$ plane is shown in blue. The five-fold modulation in the $l$ direction can be seen and the

129 peaks characteristic of this are labelled with their $(h k l)$ values. 
131 The structural solution and subsequent refinement were undertaken using the SHELXS 132 program (Sheldrick 1997), proceeding with $9 \mathrm{Fe}$ and $8 \mathrm{~S}$ atomic positions. Neutral scattering

133 factors were used for all atoms. The appropriate 3 -individual twin matrix describing a $120^{\circ}$

134 rotation along the $\mathrm{c}$ axis was introduced in the first stages of the refinement; since the three

135 individual fractions were found to be all close to $33 \%$, this step was necessary to take the

136 discrepancy factor $R 1$ below 0.2 . Individual occupancy factors for the Fe atoms were refined with

137 an overall isotropic thermal parameter to detect the partially vacant sites. The Fe sites that refined

138 to occupancies close to 1.0 were then fixed at unity, and the thermal displacement parameters were

139 then refined independently. Lastly, the ADPs were refined anisotropically, together with the

140 occupancies of the four partially vacant Fe atoms. Because of an unreasonably prolate ADP for

141 the atom at $(0,0,0) \mathrm{Fe} 9$ the thermal displacement parameters were restrained to be similar to those

142 of the neighbour Fe sites. We here highlight that this is not the same position found to be vacant

143 in the structural refinement performed by De Villiers et al. (2009), corresponding to Fe4 in our

144 model. Details of the structural solutions are given in the accompanying cif file.

145 The refinement converged with a discrepancy factor $R 1=0.0485$ for 1675 observed reflections

146 and $R 1=0.0604$ for all 2084 measured reflections and 120 parameters. The goodness of fit

147 parameter was $S=1.476$ and $w R 2$ was 0.3431 . No extinction correction parameter was used as it

148 was found to converge to zero. The largest residual electron density amounted to 1.32 e/Å.

149 Interestingly, the structural model reported by De Villiers et al. (2009) also differs in the way the

150 Fe planes stack along the [001] direction (see Figure 2). 

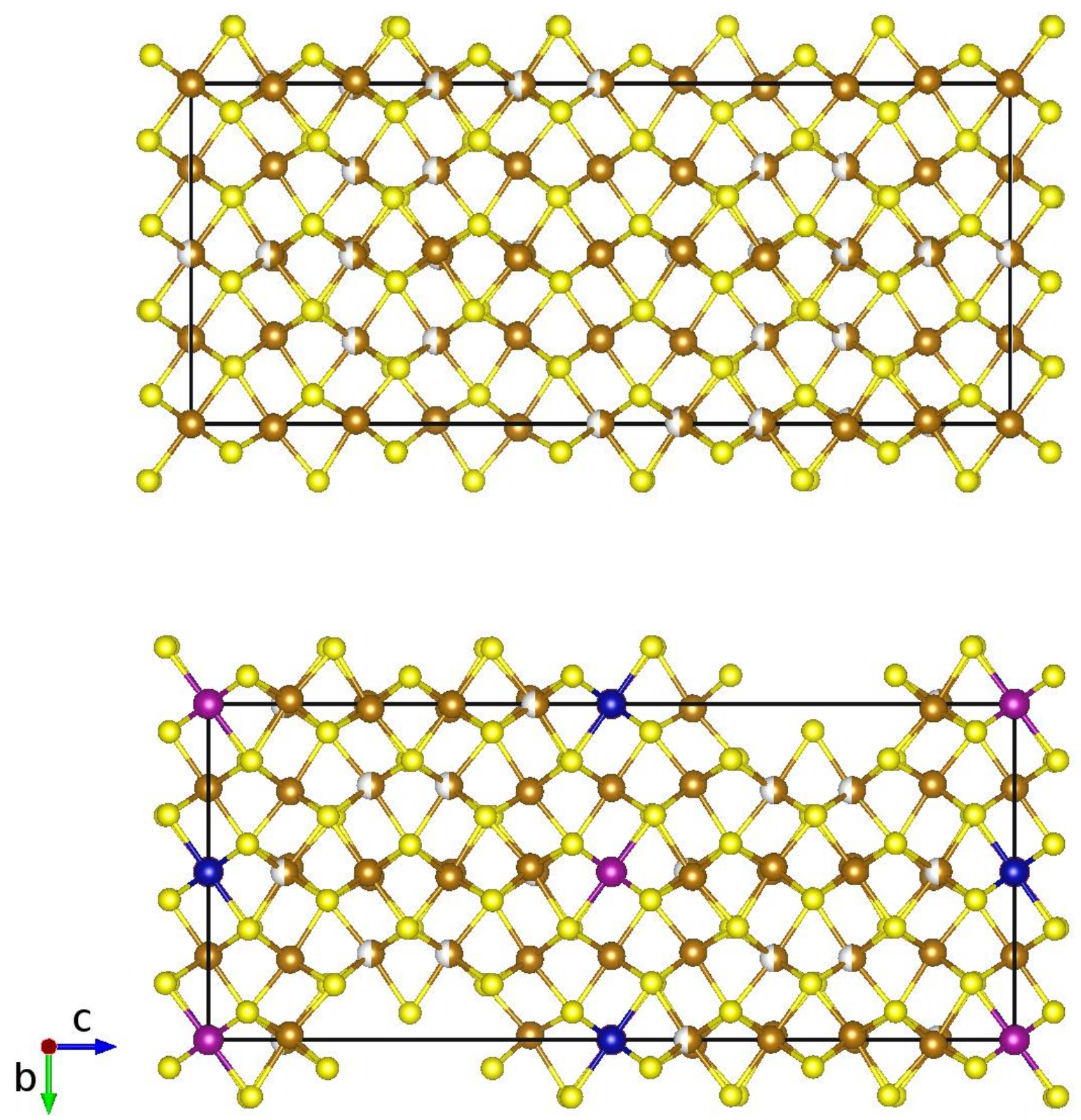

154 Figure 2. Structural model of pyrrhotite 5C viewed along [100] from this work (top) and from de

155 Villiers et al. (2009) (bottom). Color code: yellow = S sites; brown = Fe sites (partial occupancy

156 is shown by half-grey half-brown); pink = partially Ni replaced Fe site (De Villiers et al., 2009);

157 blue = vacant metal site from De Villiers et al. (2009) (see main text for details). 
159 The composition, calculated from the refinement of the structure, is $\mathrm{Fe}_{9.22(1)} \mathrm{S}_{10}$, in excellent 160 agreement with the results of the electron microprobe analysis. Details of the refinement and the

161 comparison to that of De Villiers et al. (2009) are given in Table 1. A cif file containing detailed 162 information about data collection and structural refinement is supplied separately.

163

164 Table 1. Crystal data and structure refinement details for 5C pyrrhotite.

This work

Empirical formula
Formula weight
Temperature $\left({ }^{\circ} \mathrm{K}\right)$
Wavelength $(\AA)$
Space group
Unit cell dimension

Volume

Z, Calculated Density

Absorption coefficient

$F(000)$

Crystal size

$\theta$ range for data collection

Limiting indices

Reflections collected/unique

Completeness to $\theta=25.00$

Refinement method

0.71073

$\alpha=90^{\circ}$

$\beta=90^{\circ}$

$\gamma=90^{\circ}$

12.681

3358

$\leq l \leq 42$

$0.0322]$

$99.7 \%$
De Villiers et al. (2009)

$\mathrm{Fe}_{8.79} \mathrm{Ni}_{0.118} \mathrm{~S}_{10}$

6588.86

293(2)

0.71073

Cmce (formerly Cmca)

Cmce (formerly $\mathrm{Cmca}$ )

$a=6.893(3) \AA$

$a=6.890(2) \AA$

$b=11.939(3) \AA$

$b=11.9436(4) \AA$

$c=28.635(15) \AA$

$c=28.7916(10) \AA$

$\alpha=90^{\circ}$

$\beta=90^{\circ}$

$\gamma=90^{\circ}$

2356.4(15) $\AA^{3}$

2371.36(13) $\AA^{3}$

$8,4.643 \mathrm{~g} / \mathrm{cm}^{3}$

$8,4.689 \mathrm{~g} / \mathrm{cm}^{3}$

12.516

3154

$0.14 \times 0.06 \times 0.03 \mathrm{~mm}^{3}$

$0.08 \times 0.04$ x $0.02 \mathrm{~mm}^{3}$

$2.85-26.34^{\circ}$

2.12-31.82

$-8 \leq h \leq 7,-8 \leq k \leq 14,-33 \leq$

$-9 \leq h \leq 9,-16 \leq k \leq 15,-40$

$l \leq 29$

$29278 / 2084 \quad[R($ int $)=$

$6041 / 1264[R($ int $)=0.0322]$

$99.2 \%$

Full-matrix least-squares on

Full-matrix least-squares on $F^{2}$ $F^{2}$ 
Data / restraints / parameters

Goodness-of-fit on $F^{2}$

Final $R$ indices $[I>2 \theta(I)]$

$R$ indices (all data)

Extinctions coefficient

largest diff. peak and deepest hole
2084 / 1 / 120

1.477

$R 1=0.0485, w R 2=0.3231$

$R 1=0.0604, w R 2=0.3431$

1.445 and $-1.346 \mathrm{e}^{-} \cdot \AA^{-3}$
1264 / 0 / 109

1.225

$R 1=0.0716, w R 2=0.1513$

$R 1=0.0937, w R 2=0.1627$

0.00003(2)

1.533 and $-2.125 \mathrm{e}^{-} \AA^{-3}$

\section{V. HEAT CAPACITY}

168 Heat capacity measurements were carried out in a Quantum Design PPMS. The 9.1mg sample 169 was fixed to the measurement platform of the heat capacity puck with Apiezon $\mathrm{N}$ grease. The puck

170 (plus grease) was measured through the temperature interval 2-300 K with and without the sample

171 to determine the background contribution needed to obtain the heat capacity of the sample alone.

172 At low temperatures the heat capacity of the sample was larger than the background but at 173 temperatures above $\sim 30 \mathrm{~K}$ the background was larger.

174 Figure 3 a shows a peak in the heat capacity of the crystal near $155 \mathrm{~K}$. Broad features at $215 \mathrm{~K}$

175 and $280 \mathrm{~K}$ are artefacts due to the Apiezon grease. A closer view of the peak (Fig. $3 \mathrm{~b}$ ) reveals it 176 to be at $154 \mathrm{~K}$ with an apparent onset temperature of $\sim 147 \mathrm{~K}$ and a tail up to $\sim 157 \mathrm{~K}$. Repeat 177 measurements in a field of $9 \mathrm{~T}$ applied parallel to the [100] direction of the crystal $(H / / a$ of the 178 NiAs cell) yielded a peak at $148 \mathrm{~K}$ (Fig. 3b). The shape of the peak was unchanged, but the 179 transition had clearly been shifted down in temperature by $\sim 6 \mathrm{~K}$. There is no evidence in the data 180 for the Besnus transition at $35 \mathrm{~K}$, which confirms that the crystal had fully transformed from the 181 4C structure. The peak is asymmetric. There is a shoulder on the low temperature side suggesting 182 that there are two contributions to the peak. This is somewhat similar to the heat capacity anomaly 183 recently observed in 4C pyrrhotite (Haines et al. 2019b). 

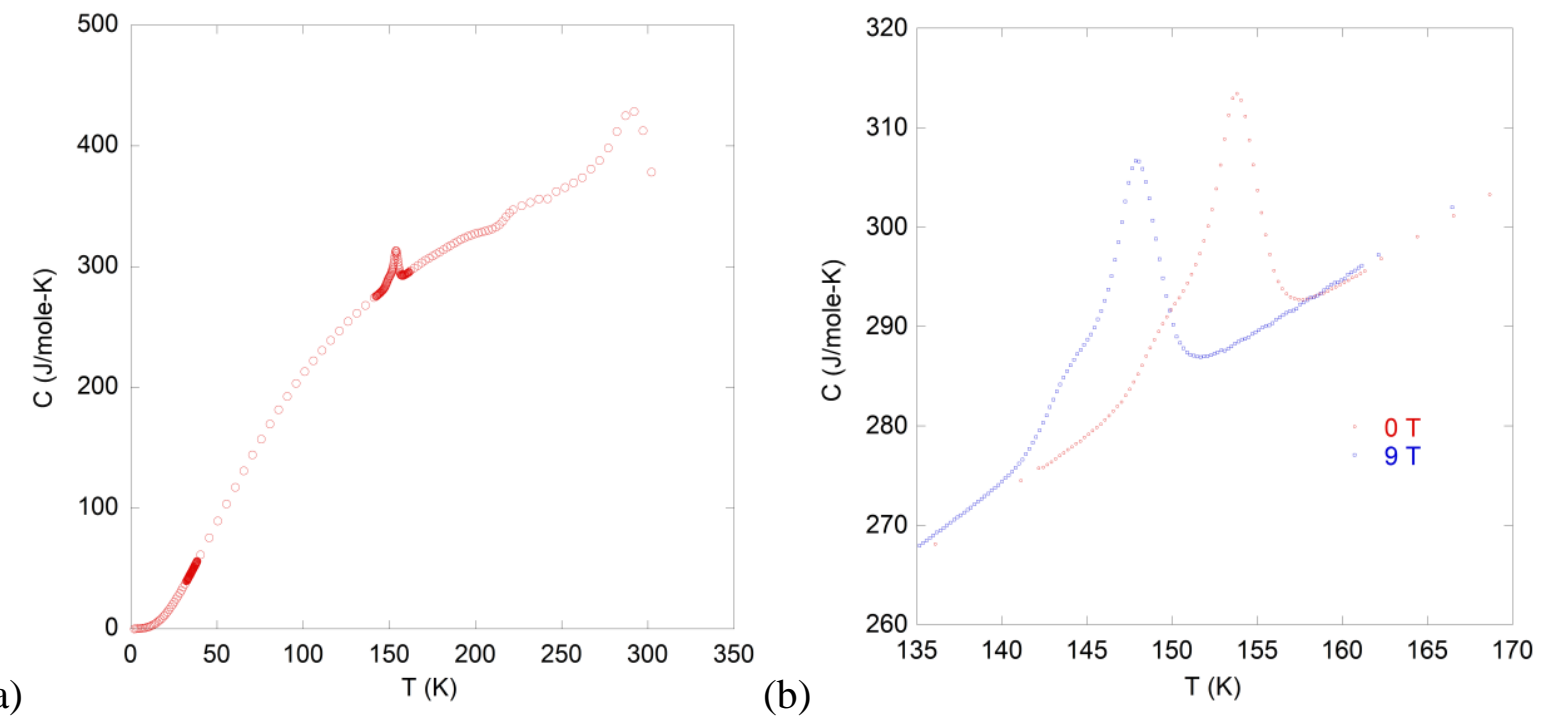

185 Figure 3. (a) Heat capacity of 5C crystal measured between 2 and $300 \mathrm{~K}$ in zero field, showing

186 the magnetic transition near $155 \mathrm{~K}$. Anomalies at $\sim 215$ and $\sim 280 \mathrm{~K}$ are artefacts. (b) Higher

187 resolution view of the peak at $154 \mathrm{~K}$ in zero field, which shifts to $148 \mathrm{~K}$ when measured in a $9 \mathrm{~T}$

188 field $(H / / a)$.

190 VI. MAGNETISATION

191 Magnetisation was measured using a Quantum Design MPMS. Zero field cooled (ZFC) data

192 were collected by cooling the crystal in zero applied field to $2 \mathrm{~K}$ before applying a $10 \mathrm{mT}$ field

$193(H / / a)$ and measuring the moment in a continuous heating sweep up to $300 \mathrm{~K}$ at $3 \mathrm{~K} / \mathrm{min}$. Field

194 cooled (FC) data were collected by cooling the crystal to $2 \mathrm{~K}$ in an applied field of $5 \mathrm{~T}$ before

195 removing the 5T field and applying a $10 \mathrm{mT}$ field $(H / / a)$ during measurements of the moment in

196 a continuous heating sweep up to $300 \mathrm{~K}$ at $3 \mathrm{~K} / \mathrm{min}$. There are two distinct features observable in

197 both the ZFC and FC magnetisation curves, at $\sim 120$ and $\sim 155 \mathrm{~K}$. The feature at $\sim 155 \mathrm{~K}$ cannot be

198 explained by any obvious impurity and matches up well with the transition seen in the heat capacity

199 of the $5 \mathrm{C}$ crystal. The feature at $\sim 120 \mathrm{~K}$ is almost certainly from the Verwey transition of 200 magnetite, revealing the presence of a small amount of magnetite as an impurity phase. This is 
201 most likely remaining from incomplete removal of the thin oxidised layer, following the heat

202 treatment at $875 \mathrm{~K}$. The same crystal was used for both magnetisation and heat capacity

203 measurements, and the lack of any anomalies in the heat capacity at $\sim 120 \mathrm{~K}$ supports the

204 conclusion that the proportion of magnetite was very small.

205 In order to put the absolute values of magnetisation in context, results for the $5 \mathrm{C}$ crystal and for

206 a 4C crystal cut from the parent crystal temperature are compared in Figure 4b. 4C pyrrhotite is

207 ferrimagnetic, due to an uncompensated moment arising from the fact that one in eight $\mathrm{Fe}$ sites are

208 vacant. The measured moment of the $4 \mathrm{C}$ crystal at room temperature is a factor of $~ 100$ larger

209 than that of the $5 \mathrm{C}$ crystal, suggesting that the magnetic ground state of $5 \mathrm{C}$ pyrrhotite is a fully

210 compensated antiferromagnet. Furthermore, this puts an upper limit on the amount of 4C

211 pyrrhotite remaining in the sample of approximately $1 \%$. In the ZFC data the step-like feature at

$212155 \mathrm{~K}$ takes place between $148 \mathrm{~K}$ and $155 \mathrm{~K}$, with a midpoint of $152 \mathrm{~K}$. The start stop and mid-

213 points of the step in the FC data are the same, though there is a small kink at the mid-point that is

214 not observed in the ZFC data.

215
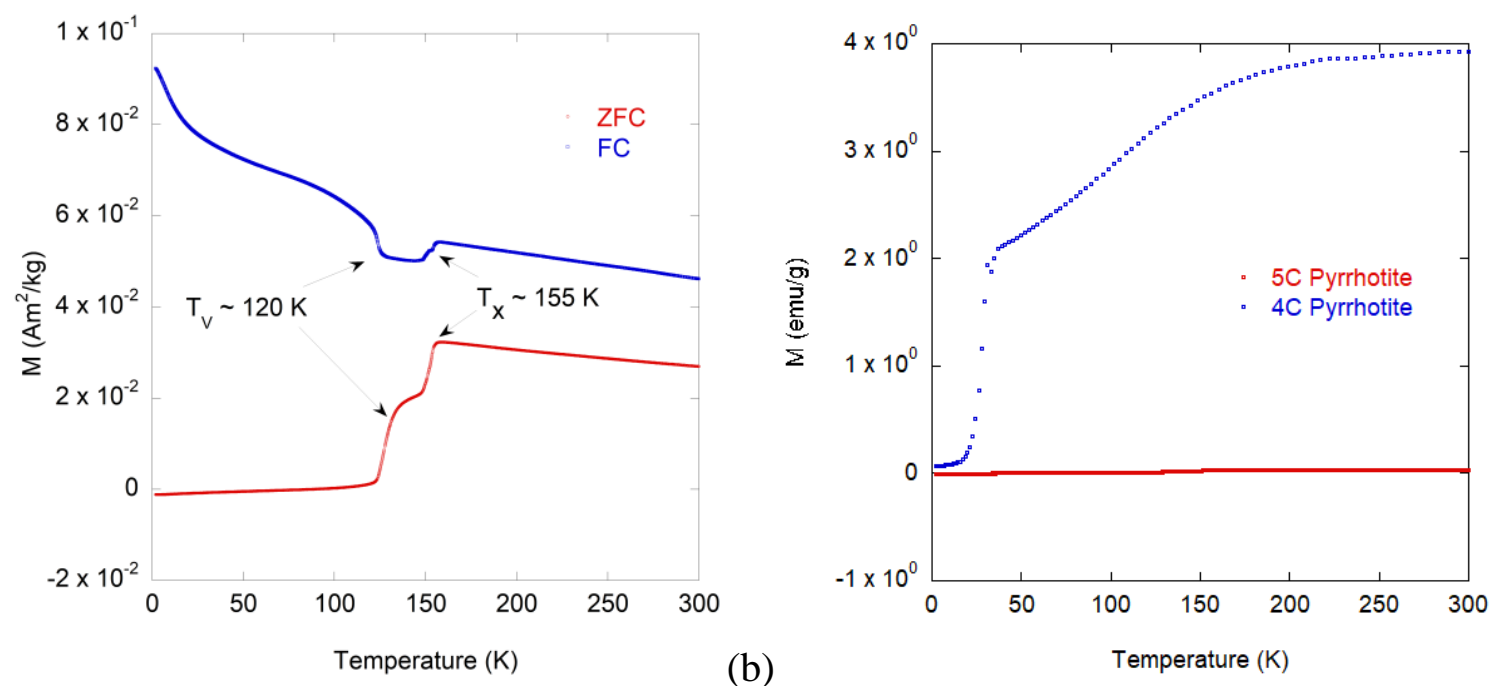

216

(a)

(b) 
217 Figure 4. Magnetisation, $M$, of 5C crystal. (a) ZFC and FC data, measured in a field of $10 \mathrm{mT}$

$218(H / / a)$. The anomaly at $\sim 120 \mathrm{~K}$ is due to the presence of a small amount of magnetite (labelled as

$219 T_{\mathrm{V}}$ for the Verwey transition) as impurity phase. The previously unknown transition is labelled as

$220 T_{\mathrm{x}}$. (b) Comparison with ZFC data for $4 \mathrm{C}$ and $5 \mathrm{C}$ crystals $(\mathrm{H} / / \mathrm{a})$, showing a difference in

221 magnetisation of a factor of $\sim 100$.

222

223 Figure 5a contains hysteresis $(M-H)$ loops measured between +7 and $-7 \mathrm{~T}$ at two temperatures

224 well above and two temperatures well below the magnetic transition. The values of $M$ never exceed

$225 \sim 2 \mathrm{Am}^{2} / \mathrm{kg}$ and are smaller at all fields by a factor of at least ten in comparison with the $4 \mathrm{C}$

226 structure (Powell et. al 2004; Charilou et. al 2015). They also show no indication of saturation at

227 any temperature, consistent with antiferromagnetic structures. Above the transition, the

228 magnetisation at $7 \mathrm{~T}\left(1.8 \mathrm{Am}^{2} / \mathrm{kg}\right)$ is more than 4 times larger than it is below the transition $(0.4$

$229 \mathrm{Am}^{2} / \mathrm{kg}$ ) at the same field. Below $150 \mathrm{~K},[100]$ is a relatively hard direction, but becomes relatively

230 soft above $\sim 150 \mathrm{~K}$. The difference remains marked even at 140 and $160 \mathrm{~K}$, but there is a

231 metamagnetic transition at $150 \mathrm{~K}$ from the low temperature magnetic structure, at low fields, to

232 the high temperature structure at high fields (Fig. 5b). This is permissive of a first order spin-flop

233 transition between two antiferromagnetic structures that have their moments aligned in the plane

234 perpendicular to the $c$-axis above the transition point and parallel to the $c$-axis below it. In this

235 scenario, small openings of the curves at low fields (Fig. 5c) and the small hysteresis seen in the

236 magnetisation curves at all temperatures are attributed to the magnetite impurity identified on the

237 basis of the magnetic anomaly at $\sim 120 \mathrm{~K}$ (Fig. 3a). In addition, the (near) saturation moment of

238 these two impurities (at a field of $1.5 \mathrm{~T}$ ) would limit their presence in the sample to similar values.

239 If the entire signal at $300 \mathrm{~K}$ and $1.5 \mathrm{~T}$ were attributed to magnetite (this is around $0.5 \mathrm{Am}^{2} / \mathrm{kg}$ ) this

240 would equate to $46 \mu \mathrm{g}$ of magnetite or $0.5 \%$ of the total mass, using a value of $90 \mathrm{Am}^{2} / \mathrm{kg}$ for 
241 saturation magnetisation of magnetite (Hunt et al. 2103 and references therein). In a similar way

242 we can constrain the $4 \mathrm{C}$ pyrrhotite to be less than $2.5 \%$ of the total mass (using $20 \mathrm{Am}^{2} / \mathrm{kg}$ as the

243 value of the magnetisation from Hunt et al. (1995) and Haines et al (2019b).

244
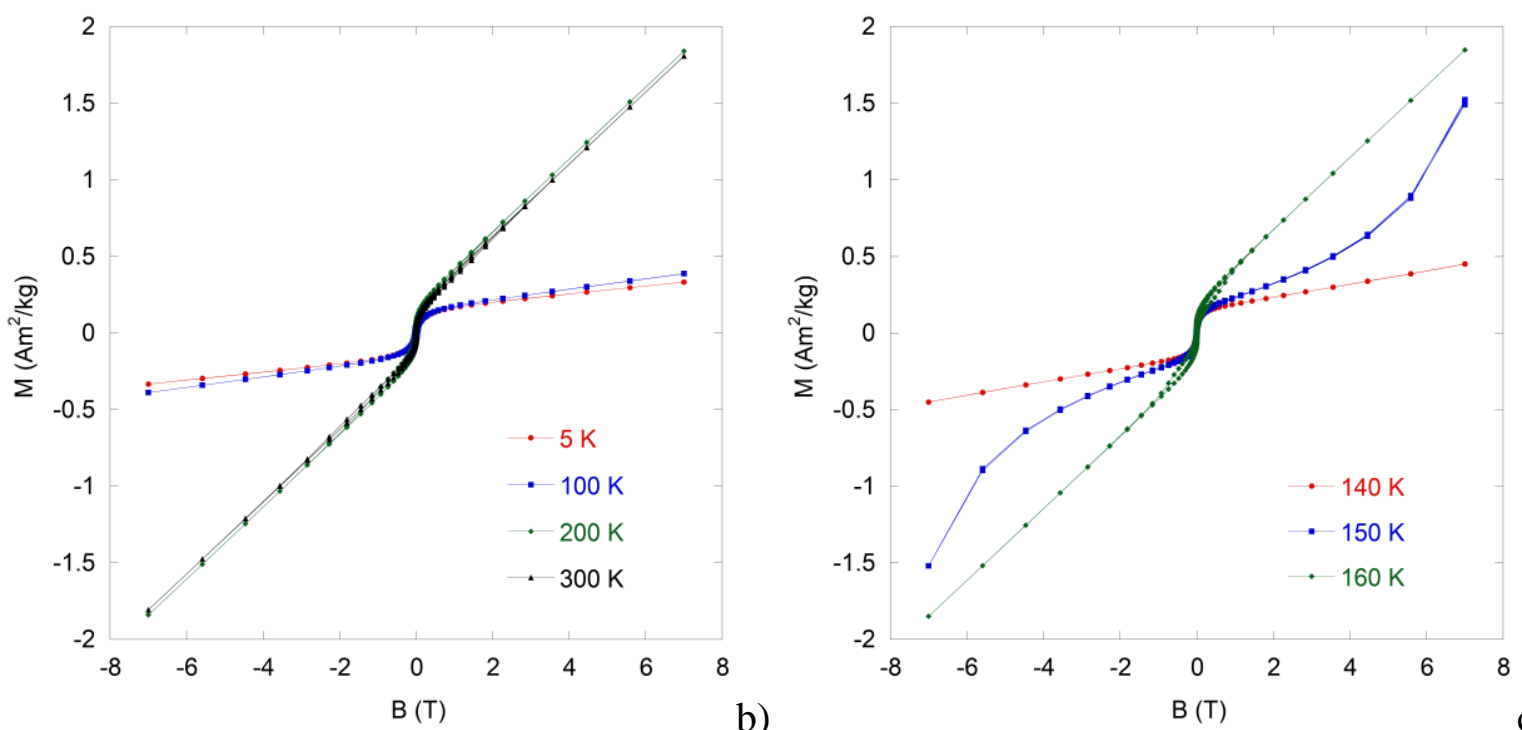

245

a)

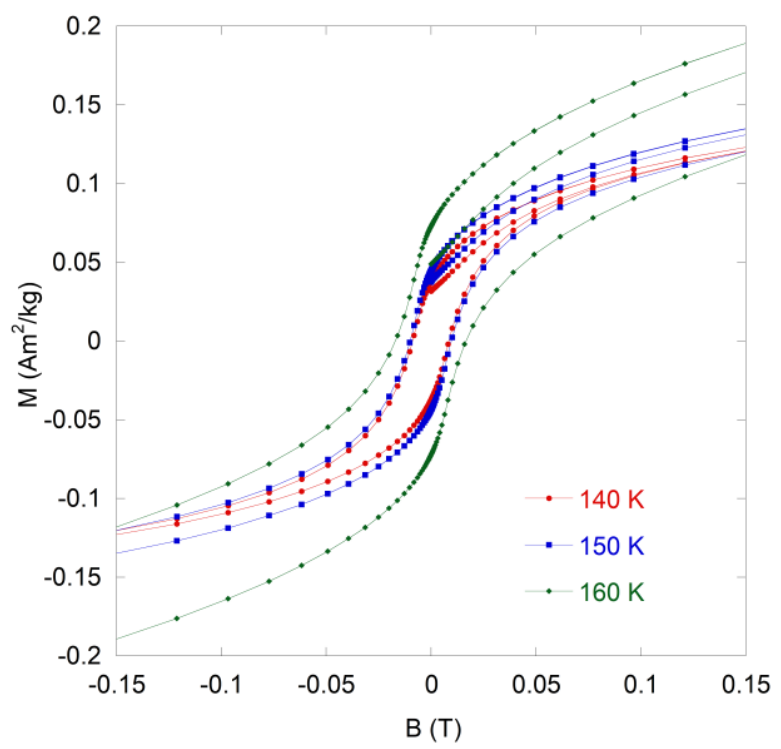

b)

c) 
250 and $100 \mathrm{~K}$. Neither show any indication of saturation at high fields. (b) Field and temperature

251 dependence of magnetisation of the $5 \mathrm{C}$ structure close to the magnetic transition at $\sim 155 \mathrm{~K}(\mathrm{H} / / \mathrm{a})$.

252 (c) Close in view of the low field region from (b), showing slight opening of the loops that is

253 attributed to the presence of a small amount of magnetite.

\section{RESONANT ULTRASOUND SPECTROSCOPY}

257 Details of the instrument used to collect RUS data at low temperatures have been given 258 elsewhere (McKnight et. al 2007). The sample sits lightly between two piezoelectric transducers

259 in the RUS head, which is attached to the end of a stick and lowered into an Orange helium flow

260 cryostat. In the present study, resonance spectra were collected in two automated cooling and

261 heating sequences with steps of $30 \mathrm{~K}$ on cooling from room temperature to $10 \mathrm{~K}$, and of between

$2621 \mathrm{~K}$ and $10 \mathrm{~K}$ on heating from $10 \mathrm{~K}$ to $295 \mathrm{~K}$. A settle time of 1200 s was included to allow for

263 thermal equilibration at each set point. Individual spectra contained 65,000 data points in the

264 frequency range $100 \mathrm{kHz}-2 \mathrm{MHz}$, with a driving voltage of $25 \mathrm{~V}$. One sequence was for a

265 different piece of the original large 4C crystal and the second was for the $9.1 \mathrm{mg}$ fragment of the

266 5C crystal. Analysis of elastic and anelastic properties was undertaken by fitting selected peaks

267 with an asymmetric Lorentzian function to give peak frequency, $f$, and width at half-maximum

268 height, $\Delta f$. The natural resonances of a $\mathrm{mm}$ sized sample involve primarily shearing motions and

269 the variations of $f^{2}$ therefore reflect variations of different combinations of predominantly shear

270 elastic constants, in this case of the single crystal. Acoustic dissipation is expressed in terms of the

271 inverse mechanical quality factor, $Q^{-1}$, which, in a RUS experiment is usually specified as $\Delta f l f$.

272 Figure 6 contains a stack of spectra from the heating sequence of the $5 \mathrm{C}$ crystal. The y-axis is

273 amplitude, but the spectra have been offset in proportion to the temperatures at which they were 
274 collected. The axis has then been relabelled as temperature in order to allow ready visualisation of 275 the principal features of the elastic behaviour. All the resonances show the same pattern of 276 reducing frequency (elastic softening) as the transition point is approached from above, followed 277 by abrupt increases (elastic stiffening) at the expected transition temperature of $\sim 155 \mathrm{~K}$. In other

278 words, all the shear elastic constants of the crystal evolve in more or less the same manner. Blue 279 curves are fits to individual peaks, and Figure 7a shows the variations of $f^{2}$ and $Q^{-1}$ for a peak near $280650 \mathrm{kHz}$. With falling temperature there is a trend of elastic softening by a few percent towards 281 the transition point, followed by $\sim 10 \%$ stiffening in a narrow temperature interval between 150 282 and $160 \mathrm{~K}$. This temperature interval corresponds almost exactly with the temperature interval 283 over which the anomaly in heat capacity extends (Fig. 3). The peaks generally remain quite sharp 284 at all temperatures, with values of $Q^{-1}$ in the vicinity of $2 \times 10^{-3}$ apart perhaps from a slight increase 285 in the vicinity of the transition point. The variation of $f^{2}$ normalised to the room temperature value 286 for three different peaks is shown in Figure 7b. Whilst the overall temperature dependence is 287 similar the peaks behave slightly differently, as would be expected for a single crystal sample in 288 which each resonance has a distinct contribution from the different elastic constants. 


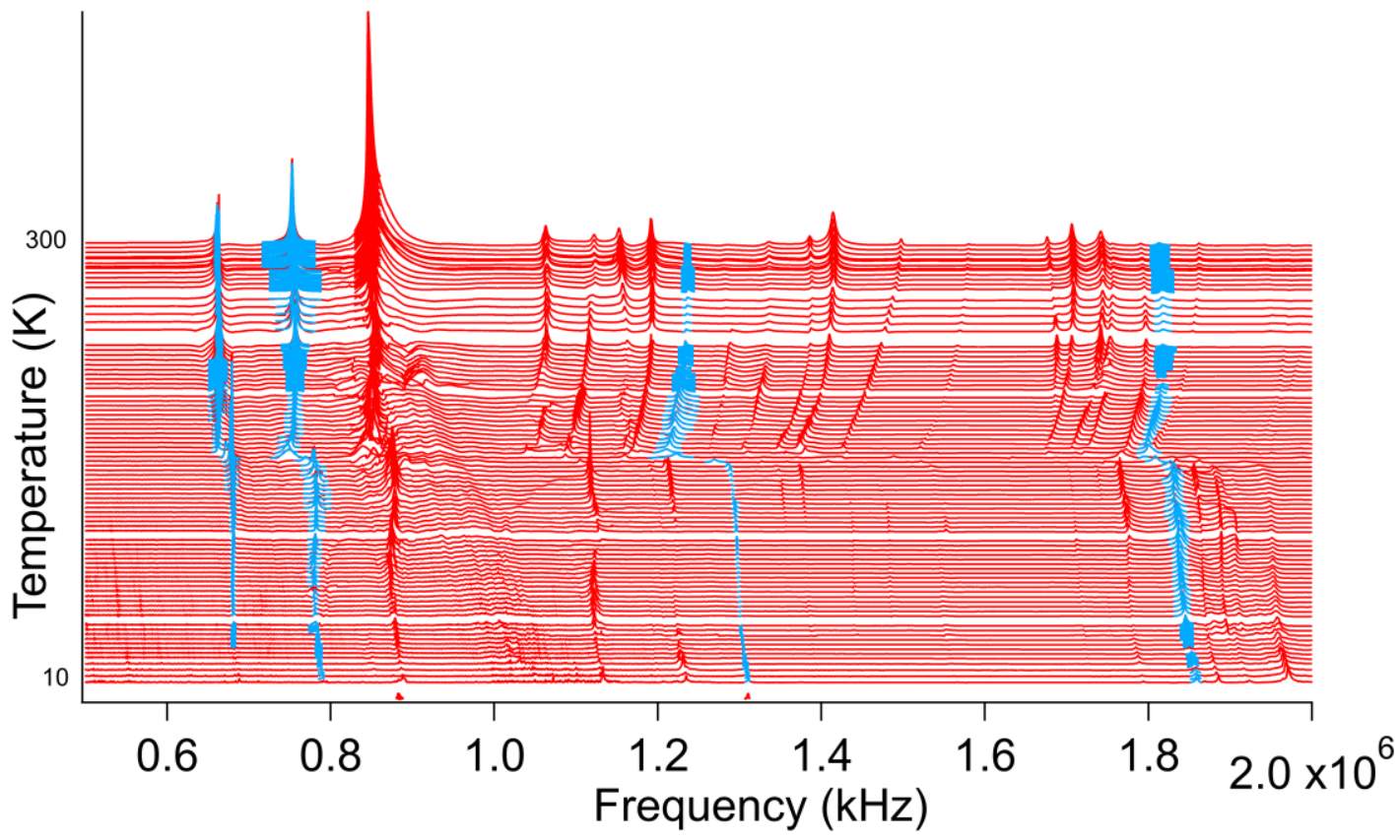

291 Figure 6. RUS spectra collected from the $9.1 \mathrm{mg}$ piece of the $5 \mathrm{C}$ crystal in a heating sequence

292 from 10 to $295 \mathrm{~K}$. The y-axis should be amplitude in volts, but the spectra have been offset in 293 proportion to the temperatures at which they were collected and the axis relabelled as temperature.

294 There is an abrupt change in frequencies of all the individual resonance peaks at the expected 295 transition temperature, $\sim 155 \mathrm{~K}$. Fits to selected peaks are shown in blue.

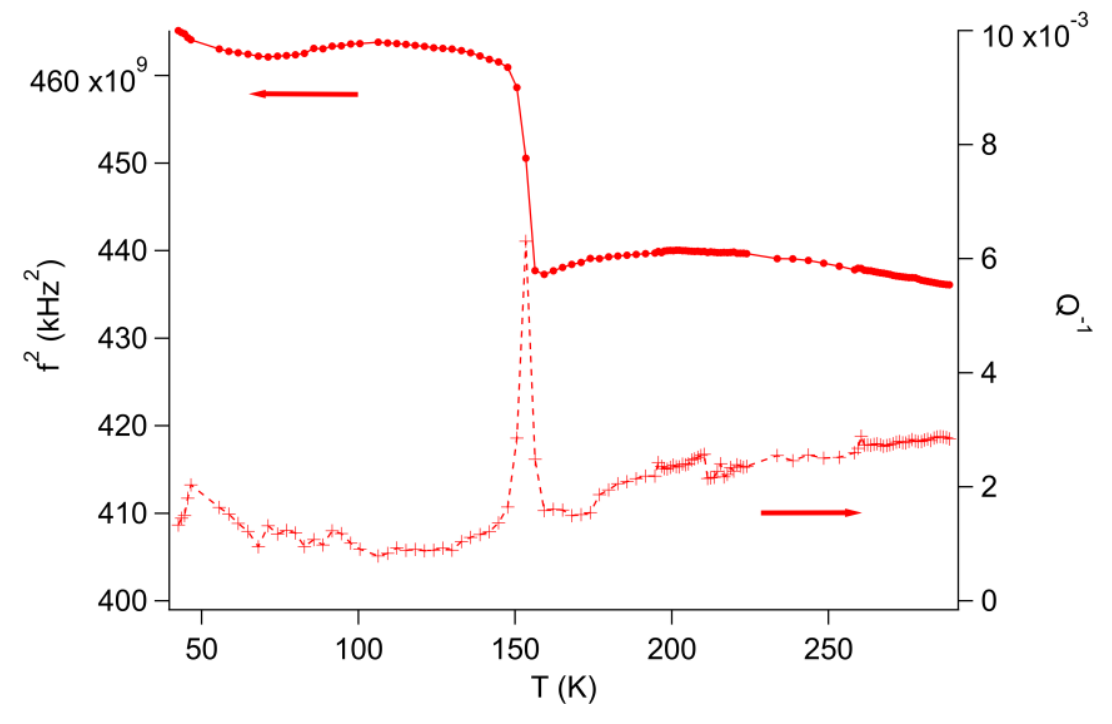


(b)
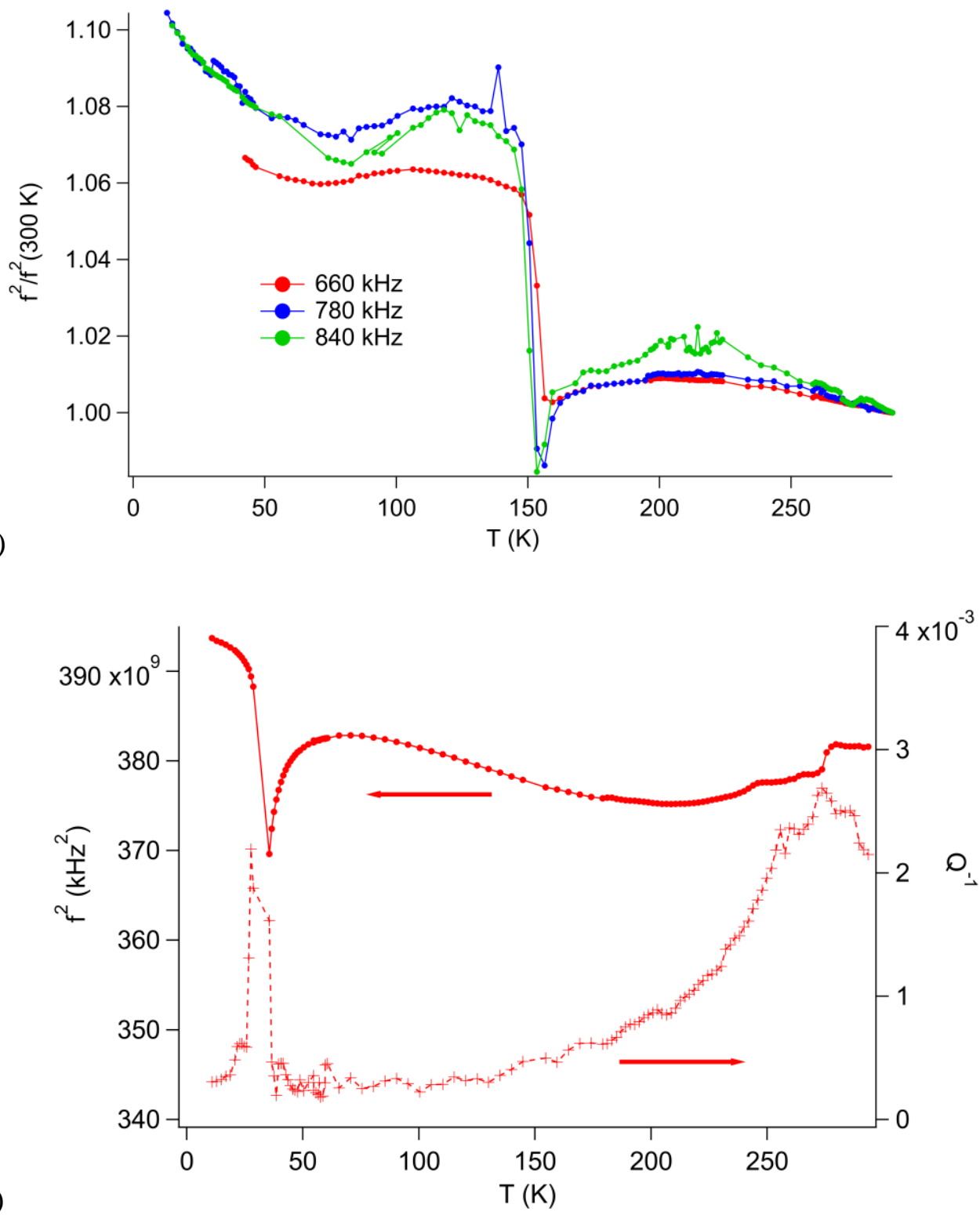

$300 \quad$ Figure 7. (a) Temperature dependence of $f^{2}$ and $Q^{-1}$ for the single crystal of $5 \mathrm{C}$ pyrrhotite from

301 fitting of the resonance peak near $650 \mathrm{kHz}$ and (b) $f^{2}$ for three resonance peaks at $660 \mathrm{kHz}, 780$

$302 \mathrm{kHz}$ and $840 \mathrm{kHz}$ normalised to the value at room temperature. (c) Temperature dependence of $f^{2}$

303 and $Q^{-1}$ for a single resonance with frequency near $620 \mathrm{kHz}$ from a $4 \mathrm{C}$ pyrrhotite crystal cut from

304 the same original sample as was used to produce the $5 \mathrm{C}$ crystal. The Besnus transition is marked

305 by a minimum if $f^{2}$ at $\sim 36 \mathrm{~K}$. Crosses $=Q^{-1}$, right axis; filled circles $=f^{2}$, left axis. 
307 Variations of $f^{2}$ and $Q^{-1}$ data from fits to a resonance peak near $620 \mathrm{kHz}$ in spectra collected

308 from the piece of original the $4 \mathrm{C}$ crystal are shown in Figure $7 \mathrm{c}$. The crystal was cut from the same

309 original sample as the piece that was converted to 5C. There are very obvious differences from the

310 variations in elastic and anelastic properties that accompany the magnetic transition shown in

311 Figure 7. In particular, softening by a few $\%$ with falling temperature is associated with a peak in

$312 Q^{-1}$ at $\sim 270 \mathrm{~K}$. This turns into a trend of gradual stiffening followed by steep softening ahead of

313 the Besnus transition, which is marked by a minimum at $\sim 36 \mathrm{~K} . f^{2}$ values then recover steeply and

314 there is a distinct peak in $Q^{-1}$ immediately below the transition. The maximum change in $f^{2}$ at the

315 frequency of this resonance, $\sim 620 \mathrm{kHz}$, is $\sim 6 \%$. Changes in elastic and anelastic properties

316 accompanying the Besnus transition in $4 \mathrm{C}$ pyrrhotite are described in detail elsewhere (Haines et

317 al 2019b).

318

319 VIII. DISCUSSION AND CONCLUSIONS

320 We have shown that $4 \mathrm{C}$ pyrrhotite can be transformed to $5 \mathrm{C}$ by annealing above the

321 concomitant Néel and vacancy ordering temperature. On the basis of single crystal x-ray

322 diffraction at room temperature we confirm that a model in space group Cmce can successfully

323 explain the diffraction data. The low temperature structure has previously been refined

324 successfully using a model in space group $P 2_{1}$ by Liles and deVilliers (2012). The Besnus

325 transition is no longer observed and a new Morin-like spin-flop transition occurs at $~ 155 \mathrm{~K}$

326 instead. The magnetic properties of the 5C crystal are incompatible with impurities of 4C

327 pyrrhotite or magnetite above the level of $1 \%$. In addition, the heat capacity anomalies

328 characteristic of the low temperature magnetic phase transitions in these two minerals were not

329 observed. The 4C crystal from which the piece used in this study was cut showed no impurities

330 above the level of detection in either powder XRD or electron microprobe measurements (Haines 
331 et al. 2019b). Therefore, it is difficult to imagine any impurity other than the magnetite formed at

332 the surface or 'untransformed' $4 \mathrm{C}$ pyrrhotite. We have carried out electron microprobe analysis

333 on the 5C crystal and the sample is monophase with a high degree of homogeneity. The impurity

334 levels are on the border of detectability. The stoichiometry is homogeneous and works out to be

$335 \mathrm{Fe}_{9.2} \mathrm{~S}_{10}$ in good agreement with the single crystal refinement.

336 The most significant structural difference in comparison with the 4C structure is the change in 337 vacancy ordering scheme which develops during cooling. Without specifying the ordering in

338 detail, it is possible to assess the changes in structure and properties from the perspective of

339 symmetry. If the reference structure at high temperatures is taken to be that of NiAs in space group

$340 P 6_{3} / m m c$, the order parameter which gives rise to the $5 \mathrm{C}$ superstructure is most likely to have the

341 symmetry of the active representation U1(1/2,0,1/5) (Haines et al 2019a). A likely candidate for

342 the magnetic order parameter has the symmetry of irreducible representation $\mathrm{m}_{5}^{+}$. The list of

343 possible magnetic space groups that can result from this combination includes the previously

344 reported crystallographic space groups $C m c e, P 2_{1} / c$ and $P 2_{1}$ (Haines et. al 2019a). New magnetic

345 and elasticity data have been interpreted here on the basis that $5 \mathrm{C}$ pyrrhotite is antiferromagnetic

346 and that the magnetic transition at $\sim 155 \mathrm{~K}$ is from one antiferromagnetic structure to another. In

347 all of the reported space groups the moments can be aligned either in the plane perpendicular to

348 the crystallographic $c$-axis of the parent or parallel to the $c$-axis. Across the $\mathrm{Fe}_{1-\mathrm{x}} \mathrm{S}$ phase diagram

349 (Schwarz and Vaughan 1972) the $590 \mathrm{~K}$ transition is to a state in which the moments are in the

350 plane perpendicular to the crystallographic $c$-axis. The low temperature spin-flop transition

351 observed in troilite and the low temperature evolution of $4 \mathrm{C}$ pyrrhotite involve a rotation of the

352 moments towards the $c$-axis.

353 Haines et. al (2019a) predicted that the magnetic transition in 5C would be an abrupt spin-flop

354 transition. This is because for none of the experimentally reported structures is it possible to have 
355 distortions which allow both $\mathrm{m}_{4}^{+}$and $\mathrm{m}_{5}^{+}$to have non-zero values. In other words, the moments

356 are strictly confined to either the plane perpendicular to the $c$-axis or to the direction parallel to

357 the $c$-axis. Cmce was reported for a crystal at room temperature with the $P 2_{1}$ structure being solved

358 using data collected at $120 \mathrm{~K}$. These two structures do not have a group-subgroup relationship so

359 that a $C m c e-P 2_{1}$ transition is necessarily first order.

360 The spin-flop transition in 5C pyrrhotite is significantly different from the Besnus transition in

361 4C pyrrhotite, as is seen clearly in differences between the two sets of elastic and anelastic data in

362 Figure 7. As a function of temperature, the spin-flop transition is characterised by a steep increase

363 in all the shear elastic constants over a narrow temperature interval, consistent with first order

364 character. The low temperature structure is stiffer than the high temperature structure and has the

365 same level of acoustic loss. In contrast, the Besnus transition occurs over a wider temperature

366 interval, consistent with a second order transition between magnetic structures that do have a

367 group-subgroup relationship. There is also a distinct peak in the acoustic loss that could relate to

368 the development of a ferroelastic microstructure. Thus, the transition in the 5C crystal appears to

369 be closely analogous to the magnetic transition to that seen in $\mathrm{FeS}$ at $440 \mathrm{~K}$, which involves the

370 same change in orientation of moments (Harihara and Murakami 1958, Andresen 1960, Sparks et.

371 al 1960 and1962, Andresen et. al 1967, Horwood et al 1976).

372 This combination of symmetry analysis and experimental data on the magnetoelastic behaviour

373 shows that 5C pyrrhotite fits well into the wider picture for the diverse structures suggested for

374 the system $\mathrm{Fe}_{1-x} \mathrm{~S}$ by Haines et al (2019a). The stable antiferromagnetic/ferromagnetic structure

375 which appears across the solid solution at $\sim 590 \mathrm{~K}$ has moments aligned perpendicular to the

376 crystallographic $c$-axis. At transition temperatures which reduce from $\sim 440 \mathrm{~K}$ with increasing

377 concentration of vacancies, this gives way to another magnetic structure in which the preferred

378 orientation of the moments is $90^{\circ}$ away from the $c$-axis. Details of the precise magnetic structure 
379 and possibilities for thermodynamically continuous pathways between the two magnetic states

380 then depend on details of the vacancy ordering at different stoichiometries, as expressed in terms

381 of order parameters with irreducible representations of the form $\mathrm{U}(1 / 2,0,1 / \mathrm{x})$ where $x$ is $3,4,5,6$

382 or irrational, corresponding to the $3 \mathrm{C}, 4 \mathrm{C}, 5 \mathrm{C}, 6 \mathrm{C}$ and incommensurate phases of pyrrhotite. All

383 these phases, apart from $3 \mathrm{C}$, are expected to show one magnetic transition below room

384 temperature. In $3 \mathrm{C}$ pyrrhotite the transition is expected to be above room temperature.

\section{IMPLICATIONS}

387 The discovery of a low temperature spin-flop transition in the $5 \mathrm{C}$ superstructure of pyrrhotite

388 must be seen within the context of the whole $\mathrm{Fe}_{1-\mathrm{x}} \mathrm{S}$ family. In this work we have shown that the

389 behaviour of the $5 \mathrm{C}$ superstructure is consistent with a comprehensive group theoretical

390 framework described in Haines et al. (2019a). The result fits with the 3C superstructure being

391 ferrimagnetic at room temperature and, if the crystallographic space group of $\mathrm{P} 3_{1} 21$ (Nakano et

392 al. 1979; Keller-Besrest et al. 1983) is correct, with the magnetic moments aligned parallel to the

$393 c$-axis. A low temperature spin-reorientation transition like the Besnus transition must also be

394 expected in the 6C superstructure. Thus, it seems that the spin flop transitions are all closely

395 related and fundamentally due to the same underlying thermodynamic driving force.

396 An additional consideration relates to the fact that the 5C crystal studied in this work was the

397 result of thermally cycling a 4C crystal through the concomitant vacancy ordering and

398 ferrimagnetic ordering temperature, at $595 \mathrm{~K}$, and then up to $875 \mathrm{~K}$ before cooling back to room

399 temperature. To our knowledge, this is the first time that $5 \mathrm{C}$ pyrrhotite has been synthesised in 400 this way.

401 Finally, our improved understanding of the nature of the vacancy ordering and related magnetic 402 structures in the $\mathrm{Fe}_{1-\mathrm{x}} \mathrm{S}$ system means that an understanding of the microscopic mechanisms may 
403 now be within reach for an important group of mineral sulphides. Further experiments and

404 calculations should focus on the changes in electronic structure behind the changing magnetic

405 anisotropy and its dependence on temperature, pressure and vacancy order.

407 ACKNOWLEDGMENTS

408 The authors acknowledge funding from the Leverhulme Foundation, grant number RPG2016-

409 298. Heat capacity and DC magnetic measurements were carried out using the Advanced Materials

410 Characterisation Suite, funded by EPSRC Strategic Equipment Grant EP/M000524/1. RUS

411 facilities in Cambridge were funded by grants to MAC from the Natural Environment Research

412 Council of Great Britain (grant nos. NE/B505738/1 and NE/F17081/1) and from the Engineering

413 and Physical Sciences Research Council (grant no. EP/I036079/1).

414

\section{$415 \quad$ References}

416 Andresen, A. F., Hofman-Bang, N., Bak, T. A., Varde, E. \& Westin, G. (1960). Acta Chemica

417 Scandinavica, 14, 919-926.

418 Andresen, A. F., Torbo, P., Ostlund, E., Bloom, G. \& Hagen, G. (1967). Acta Chemica Scandinavica, 21, 419 2841-2848.

420 Bertaut, E. F. (1953). Acta Crystallographica, 6, 557-561

421 Besnus, M. J. \& Meyer, A. J. P. (1964) Proceedings of the International Conference on Magnetism

422 Nottingham 507-511

423 Charilaou, M., Kind, J., Koulialias, D., Weidler, P. G., Mensing, C., Löffler, J. F. \& Gehring. A. U.

424 (2015). Journal of Applied Physics, 118083903

425 CrysAlisPro Software System, Rigaku Oxford Diffraction, (2018).

426 De Villiers, J. P. R. \& Liles, D. C. \& Becker, M. (2009). American Mineralogist, 94(10), 1405-1410.

427 De Villiers, J. P. R. \& Liles, D. C. (2010). American Mineralogist, 95(1), 148-152. 
428 Elliot, A. D. (2010). Acta Crystallographica Section B, 66(3), 271-279.

429 Fleet, M. E. (1971). Acta Crystallographica Section B, 27(10), 1864-1867.

430 Haines, C. R. S., Howard, C. J., Harrison, R. J. and Carpenter, M. A. (2019a) Acta Crystallographica

431 Section B 75, 1208-1224

432 Haines, C. R. S., Volk, M., Dutton, S. E. and Carpenter, M. A. (2019b) in preparation

433 Haines, C. R. S., Lampronti, G. I.. and Carpenter, M. A. (2019c) in preparation

434 Horwood, J. L., Townsend, M. G. \& Webster, A. H. (1976) Journal of Solid State Chemistry 17 17-42.

435 Hunt, C. P., Moskowitz, B. M. and Banerjee, S. K. (2013). Magnetic Properties of Rocks and Minerals.

436 In Rock Physics \& Phase Relations, T. J. Ahrens (Ed.). doi:10.1029/RF003p0189

437 Keller-Besrest, F., Collin, G. \& Comes, R. (1983). Acta Crystallographica Section B 39, 296-303.

438 Kontny, A., De Wall, H., Sharp, T., \& Posfai, M. (2000). The American Mineralogist, 85(10), 1416-

4391427.

440 Koto, K., Morimoto, N., \& Gyobu, A. (1975). Acta Crystallographica Section B, 31(12), 2759-2764.

441 Liles, D. C. \& De Villiers, J. P. R. (2012). American Mineralogist, 97(2), 257-261.

442 Martín-Hernández, F., Dekkers, M., Bominaar-Silkens, I., \& Maan, J. (2008). Geophysical Journal

443 International, 174(1), 42-54.

444 Mcknight, R., Carpenter, M., Darling, T., Buckley, A., \& Taylor, P. (2007). American Mineralogist, 445 92(10), 1665-1672.

446 Morimoto, N., Gyobu, A, Tsukuma, K, \& Koto, K. (1975). The American Mineralogist., 60(3-4), 240447248.

448 Nakano, A., Tokonami, M. \& Morimoto, N. (1979). Acta Crystallographica Section B, 35(3), 722-724.

449 Nakazawa, H. \& Morimoto, N. (1971). Material Research Bulletin, 6 345-358.

450 Posfai, M., Sharp, T., \& Kontny, A. (2000). The American Mineralogist, 85(10), 1406-1415.

451 Powell, A., Vaqueiro, P., Knight, K., Chapon, L. \& Sanchez, R. (2004). Physical Review B, 70(1).

452 Rochette, P., Gattacceca, J., Chevrier, V., Hoffmann, V., Lorand, J., Funaki, M., \& Hochleitner, R. 453 (2005). Meteoritics \& Planetary Science, 40(4), 529-540. 
454 Schwarz, E. J. \& Vaughan, D. J., (1972). Magnetic Phase Relations of Pyrrhotite. Journal of

455 Geomagnetism and Geoelectricity. 24. 441-458. DOI:10.5636/jgg.24.441.

456 Sheldrick, G. M. (1997). shelx197 and shelxs97. University of Göttingen, Germany.

457 Sheldrick, G.M., Acta Crystallographica Section C (2015), C27, 3-8.

458 Sparks, J. T., Mead, W., Kirschbaum, A. J. \& Marshall, W. (1960). Journal of Applied Physics, 31(5),

459 S356-S357.

460 Sparks, J. T., Mead, W. \& Komoto, T. (1962) Journal of the Physical Society of Japan 17(Suppl. B-I)

$461 \quad 249-252$.

462 Yamamoto, A. \& Nakazawa, H. (1982). Acta Crystallographica Section A, 38(1), 79-86. 